\title{
ON VARIATIONAL INEQUALITIES IN SEMI-INNER PRODUCT SPACES
}

\author{
MUHAMMAD ASLAM NOOR
}

\begin{abstract}
A bstract. In this paper, we consider and study the variational inequalities in the setting of semi-inner product spaces. Using the auxiliary principle, we propose and analyze an innovative and novel iterative algorithm for finding the approximate solution of variational inequalities. We also discuss the convergence criteria.
\end{abstract}

\section{Introduction}

Variational inequality theory has been used to study a wide class of free, moving and equilibrium problems arising in various branches of pure and applied science in a general and unified framework. This theory has been extended and generalized in several directions using new and powerful methods that have led to the solution of basic and fundamental problems thought to be inaccessible previously. Much work in this field has been done either in inner product spaces or in Hilbert spaces and it is generally thought that this is desirable, if not essential, for the results to hold. We, in this paper, consider and study the variational inequalities in the setting of semi-inner product spaces, which are more general than the inner product space. It is observed that the projection technique can not be applied to show that the variational inequality problem is

Received January 6, 1991; revised March 20, 1992.

(1980) AMS(MOS) Subject Classifications: 49J40, 65K05.

Keywords and phrases: Variational inequalities, Auxiliary principle, Iterative

algorithms, Semi-inner product spaces. 
equivalent to a fixed point problem in semi-inner product spaces. This motivates us to use the auxiliary principle technique to suggest and analyze an iterative algorithm to compute the approximate solution of variational inequalities. In Section 2, we review some basic results and formulate the problems. Main results are discussed in Section 3.

\section{Basic Results and Formulations}

A real vector space $H$ is said to be a semi-inner product space, if there is a function $\langle\cdot, \cdot\rangle: H \times H \rightarrow R$ with the following properties for all $x, y, z \in H$ and $\mu, \lambda \in R$

(i) $<x, x>>0, \quad$ for $x \neq 0$.

(ii) $\langle\lambda x+\mu y, z\rangle=\lambda\langle x, z\rangle+\mu\langle y, z\rangle$

(iii) $|\langle x, y\rangle|^{2} \leq\langle x, x\rangle\langle y, y\rangle$.

It was Lumer [5], who originally introduced and studied the semi-inner product spaces. We also note that a semi-inner product space is a semi-normed linear space with norm $\|x\|^{2}=\langle x, x\rangle$. It has been shown [5] that a normed linear space can be made a semi-inner product space in a unique way if and only if it is smooth. In general, every normed linear space can be made a semi-inner product space in infinitely many different ways. Giles [2] has proved that if $H$ is uniformly convex smooth Banach space, then the semi-inner product has the following properties.

(a) $\langle x, \lambda y\rangle=\lambda\langle x, y\rangle$, for all $x, y \in H, \lambda \in R$

(b) $\langle x, y\rangle=0$ if and only if $y$ is orthogonal to $x$.

For further properties and applications, see $[2,5]$.

Let $K$ be a closed convex set in a semi-inner product space $H$. Given a continuous mapping $T$ from $K$ into $H$, we consider the problem of finding $u \in \mathbb{K}$ such that

$$
<T u, v-u>+b(u, v)-b(u, u) \geq 0,
$$

where the form $b(\cdot, \cdot): H \times H \rightarrow R$ is a nondifferentiable and satisfies the following properties: 
(i) $b(u, v)$ is linear in the first argument.

(ii) $b(u, v)$ is a bounded form on $H \times H$, that is, there exists a constant $\gamma>0$ such that

$$
|b(u, v)| \leq \gamma\|u\|\|v\|, \quad \text { for all } u, v \in H .
$$

(iii) $b(u, v)-b(u, w) \leq b(u, v-w)$

(iv) $b(u, v)$ is convex in the second argument.

We remark that the problem (2.1) appears to be new one in the setting of the semi-inner product space. In a Hilbert space, the problem (2.1) has been studied by many authors including Kikuchi and Oden [4] and Noor [6] using quite different techniques. We also note that if $T$ is a linear symmetric positive operator and $b(u, v)$ satisfies the properties (i)-(iv), then one can easily show that the problem (2.1) is equivalent to finding the minimum of the functional $I[v]$ on $K$ in $H$, where

$$
I[v]=1 / 2<T v, v>+b(v, v) .
$$

We also need the following concepts.

Definition 2.1. An operator $T: H \rightarrow H$ is said to:

(a) Strongly monotone, if there exists a constant $\alpha>0$ such that

$$
<T u-T v, u-v>\geq \alpha\|u-v\|^{2}, \quad \text { for all } u, v \in H \text {. }
$$

(b) Lipschitz continuous, if there exists a constant $\beta>0$ such that

$$
\|T u-T v\| \leq \beta\|u-v\|, \quad \text { for all } u, v \in H \text {. }
$$

In particular, it follows that $\alpha \leq \beta$.

\section{Main Results}

We note that the projection technique cannot be used to study the existence of a solution of problem (2.1) in the semi-inner product space. We, here, use the 
auxiliary principle technique of Noor [6] and Glowinski, Lions and Tremolieres [3] to prove the existence of a solution of the variational inequality (2.1) and suggest an innovative algorithm to compute the approximate solution of the variational inequalities.

Theorem 3.1. Let T be a strongly monotone Lipschitz continuous operator. If the form $b(u, v)$ satisfies the conditions (i)-(iii) and is positive, then there exists a unique solution $u \in K$ satisfying (2.1).

\section{Proof.}

(a) Uniqueness: Let $u_{1} \neq u_{2} \in K$ be two solutions of (2.1), then for all $v \in K$, we have

$$
\begin{aligned}
& <T u_{1}, v-u_{1}>\geq b\left(u_{1}, u_{1}\right)-b\left(u_{1}, v\right) \\
& <T u_{2}, v-u_{2}>\geq b\left(u_{2}, u_{2}\right)-b\left(u_{2}, v\right) .
\end{aligned}
$$

Now taking $v=u_{2}$ in (3.1) and $v=u_{1}$ in (3.2), adding the resultant inequalities and using the positivity of the form $b(u, v)$, we obtain

$$
<T u_{1}-T u_{2}, u_{1}-u_{2}>\geq b\left(u_{2}-u_{1}, u_{1}-u_{2}\right) \leq 0
$$

Since $T$ is a strongly monotone, so there exists a constant $\alpha>0$ such that

$$
\alpha\left\|u_{1}-u_{2}\right\|^{2} \leq<T u_{1}-T u_{2}, u_{1}-u_{2}>\leq 0,
$$

from which it follows that $u_{1}=u_{2}$, the uniqueness of the solution of (2.1).

(b) Existence: We now use the auxiliary principle technique to prove the existence of the solution of (2.1). For a given $u \in K$, we consider the auxiliary problem of finding a unique $w \in K$, see [3, Page 15-16] such that

$$
<w, v-w>+\rho b(u, v)-\rho b(u, w) \geq\langle u, v-w>-\rho<T u, v-w\rangle
$$

for all $v \in K$ and $\rho>0$ is a constant. 
Let $w_{1}, w_{2}$ be two solutions of (3.3) related to $u_{1}, u_{2} \in \mathbb{K}$ respectively. It is enough to show that the mapping $u \rightarrow w$ has a fixed point belonging to $K$ satisfying (3.3). In other words, it is enough to show that

$$
\left\|w_{1}-w_{2}\right\| \leq \theta\left\|u_{1}-u_{2}\right\|
$$

with $0 \leq \theta<1$, where $\rho>0$ is a constant. Taking $v=w_{2}$ (respectively $w_{1}$ ) in (3.3) related to $u_{1}$ (respectively $u_{2}$ ) and adding the inequalities, we have $<w_{1}-w_{2}, w_{1}-w_{2}>\leq \rho b\left(u_{1}-u_{2}, w_{2}-w_{1}\right)+<u_{1}-u_{2}-\rho\left(T u_{1}-T u_{2}\right), w_{1}-w_{2}>$, from which, using (2.2), it follows that

$$
\left\|w_{1}-w_{2}\right\| \leq\left\|u_{1}-u_{2}-\rho\left(T u_{1}-T u_{2}\right)\right\|+\rho \gamma\left\|u_{1}-u_{2}\right\|,
$$

where $\gamma$ is the boundedness constant of the form $b(u, v)$.

Since $T$ is a strong monotone Lipschitz continuous operator, so

$$
\begin{aligned}
\left\|u_{1}-u_{2}-\rho\left(T u_{1}-T u_{2}\right)\right\|^{2} & \leq\left\|u_{1}-u_{2}\right\|^{2}-2 \rho<T u_{1}-T u_{2}, u_{1}-u_{2}>+\rho^{2}\left\|T u_{1}-T u_{2}\right\|^{2} \\
& \leq\left(1-2 \alpha \rho+\rho^{2} \beta^{2}\right)\left\|u_{1}-u_{2}\right\|^{2} .
\end{aligned}
$$

From (3.4) and (3.5), we obtain

$$
\left\|w_{1}-w_{2}\right\| \leq \theta\left\|u_{1}-u_{2}\right\|
$$

where $\theta=\left(\sqrt{1-2 \alpha \rho+\beta^{2} \rho^{2}}+\gamma \rho\right)<1$ for $0<\rho<\frac{2(\alpha-\gamma)}{\beta^{2}-\gamma^{2}}, \gamma<\alpha$ and $\rho \gamma<1$, showing that the mapping $u \rightarrow w$ defined by (3.3) has a fixed point, which is the solution of (2.1), the required result.

Remark 3.1. It is clear that for each $u \in K$ and $\rho>0, w \in \mathbb{K}$ satisfying (3.3) is equivalent to finding the minimum of the functional $F[v]$, where

$$
F[v]=1 / 2\langle v, v\rangle+\left\langle\rho T u-{ }_{u} u, v>+\rho b(u, v),\right.
$$

which is a convex functional associated with the variational inequality (3.3). Following the ideas of Cohen [1] and Noor [6], we propose and analyze a general algorithm. 
For some $u \in \mathbb{K}$, we introduce the following general auxiliary problem of finding the minimum of the functional $J[w]$ on $K$ in $H$, where

$$
J[w]=E(w)+<\rho T(u)-E^{\prime}(u), w>+\rho b(u, w) .
$$

Here $E(w)$ is a convex differentiable functional and $\rho>0$ is a constant. If the bilinear form $b(u, v)$ is positive and satisfies the conditions (i)-(iv), one can easily show that the minimum of $J[w]$ on $K$ can be characterized by an auxiliary variational inequality

$$
<E^{\prime}(w), v-w>+\rho b(u, v)-\rho b(u, w) \geq<E^{\prime}(u), v-w>-\rho<T u, v-w>
$$

for all $v \in \mathbb{K}$.

It is obvious that the auxiliary variational inequality (3.3) is a special case of (3.7). We also note that if $w=u$, then $w$ is a solution of the variational inequality (2.1). It is well known that in many applications the auxiliary variational inequalities (3.3) and (3.7) occur, which do not arise as a result of minimization problems. This motivates the interest of studying problems (3.3) and (3.7) on its own, that is, without assuming a priori that these come out as an Euler inequality of an extremum problem. The main motivation of this paper is to suggest a general auxiliary variational inequality problem, which includes (3.3) and (3.7) as special cases.

Auxiliary Problem 3.1. For some $u \in \mathbb{K}$, we consider the general auxiliary variational inequality problem of finding $w \in \mathbb{K}$ such that

$$
<A(w), v-w>+\rho b(u, v)-\rho b(u, w) \geq<A(u), v-w>-\rho<T u, v-w>
$$

for all $v \in K$, wher $\rho>0$ is a constant and $A$ is a nonlinear operator on $H$.

It is obvious that if $w=u$, then $w$ is a solution of the variational inequality (2.1). Based on these observations, we now suggest and analyze the following algorithm for variational inequalities in the semi-inner product spaces $H$. 


\section{Algorithm 3.1.}

(a) At $n=0$, start with some initial $w_{0}$.

(b) At step $n$, solve the auxiliary problem (3.8) with $u=w_{n}$. Let $w_{n+1}$ denote the solution of the problem (3.8).

(c) If $\left\|w_{n+1}-w_{n}\right\| \leq \varepsilon$, for given $\varepsilon>0$, stop. Otherwise repeat (b).

We now study those conditions under which the approximate solution $w_{n}$ obtained from Algorithm 3.1 converges to the exact solution $w$ of (2.1).

Theorm 3.2. Let $w_{n}$ and $w$ be the solution of (3.8) related to $u_{n}, u \in K$ respectively. If the operators $T$ and $A$ are both strongly and Lipschitz continuous respectively and the form $b(u, v)$ satisfies the conditions (i)-(iii), then

$$
w_{n} \rightarrow w
$$

for

$$
\begin{aligned}
\left|\rho-\frac{\alpha-\mu \gamma}{\beta^{2}-\gamma^{2}}\right| & <\left\{(\alpha-\mu \gamma)^{2}-\left(\beta^{2}-\gamma^{2}\right)\left(1-\mu^{2}\right)\right\}^{1 / 2} /\left(\beta^{2}-\gamma^{2}\right), \rho \gamma<\mu \\
\alpha & >\mu \gamma+\sqrt{\left(\beta^{2}-\gamma^{2}\right)\left(1-\mu^{2}\right)} .
\end{aligned}
$$

Proof. Since $w_{n}$ and $w$ are the two solutions of (3.8) related to $u_{n}, u \in K$ respectively. In order to prove the convergence of the approximate solution $w_{n}$ to the exact solution $w$ of (3.8), it is sufficient to show that for $\rho>0$ well chosen, $\left\|w_{n}-w\right\| \leq \theta\left\|u_{n}-u\right\|$. Now taking $v=w$ (respectively $w_{n}$ ) in (3.8) related to $u_{n}$ (respectively $u$ ), adding the resultant inequalities and using (iii), we obtain

$$
\begin{aligned}
< & A\left(w_{n}\right)-A(w), w_{n}-w> \\
\leq< & A\left(u_{n}\right)-A(u), w_{n}-w>+\rho b\left(u_{n}-u, w_{n}-w\right) \\
& -\rho<T^{\prime} u_{n}-T u, w_{n}-w> \\
= & <A\left(u_{n}\right)-A(u)-\left(u_{n}-u\right), w_{n}-w>+\rho b\left(u_{n}-u, w_{n}-w\right) \\
& +<u_{n}-u-\rho\left(T u_{n}-T u\right), w_{n}-w>.
\end{aligned}
$$

Now using the strongly monotonicity and Lipschitz continuity of the operators 
$T$ and $A$, and using (2.2), we have

$$
\begin{aligned}
& n\left\|w_{n}-w\right\|^{2} \\
\leq & \left\|u_{n}-u-\left(A\left(u_{n}\right)-A(u)\right)\right\|\left\|w_{n}-w\right\|+\rho \gamma\left\|u_{n}-u\right\|\left\|w_{n}-w\right\| \\
& +\left\|u_{n}-u-\rho\left(T u_{n}-T u\right)\right\|\left\|w_{n}-w\right\| \\
\leq & \left\{\left(\sqrt{1-2 \eta+\xi^{2}}\right)+\rho \gamma+\sqrt{1-2 \alpha \rho+\rho^{2} \beta^{2}}\right\}\left\|u_{n}-u\right\|\left\|w_{n}-w\right\|,
\end{aligned}
$$

where $\xi$ is the Lipschitz constant and $\eta$ is the strongly monotonicity constant of $A$ respectively. Hence,

$$
\left\|w_{n}-w\right\| \leq\left\{\frac{k+\rho \gamma+t(\rho)}{n}\right\}\left\|u_{n}-u\right\|=\theta\left\|u_{n}-u\right\|,
$$

where $\theta=\frac{k+\rho \gamma+t(\rho)}{n}, k=\sqrt{1-2 \eta+\xi^{2}}$ and $t(\rho)=\sqrt{1-2 \alpha \rho+\beta^{2} \rho^{2}}$.

We have to show that $\theta<1$. It is clear that $t(\rho)$ assumes its minimum value for $\bar{\rho}=\frac{\alpha}{\beta^{2}}$ with $t(\bar{\rho})=\sqrt{1-\frac{\alpha^{2}}{\beta^{2}}}$. For $\rho=\bar{\rho}, \frac{k+\rho \gamma+t(\rho)}{n}<1$ with $\mu=n-k>0$ implies that $\rho \gamma<\mu$. Thus it follows that $\theta<1$ for all $\rho$ with

$$
\begin{gathered}
\left|\rho-\frac{\alpha-\mu \gamma}{\beta^{2}-\gamma^{2}}\right|<\left\{(\alpha-\mu \gamma)^{2}-\left(\beta^{2}-\gamma^{2}\right)\left(1-\mu^{2}\right)\right\}^{1 / 2} /\left(\beta^{2}-\gamma^{2}\right), \\
\rho \gamma<\mu \text { and } \alpha>\mu \gamma+\sqrt{\left(\beta^{2}-\gamma^{2}\right)(1-\mu)^{2}} .
\end{gathered}
$$

Since $\theta<1$, so the approximate solution $w_{n}$ converges to $w$, the exact solution of (3.8) strongly in $H$, the required result.

\section{Acknowledgement}

The author would like to thank the referee for his useful suggestions and comments.

\section{References}

[1] G. Cohen, "Auxiliary problem principle extended to variational inequalities", J. Optim. Theor. Appl. 59 (1988), 325-33.

[2] R. Giles, "Classes of semi-inner product spaces", Trans. Amer, Math. Soc. 129 (1967), 436-466. 
[3] R. Glowinski, J. L. Lions and R. Tremolieres, "Numerical Analysis of Variational Inequalities", North-Holland, Amsterdam, 1981.

[4] N. Kikuchi and J. T. Oden, "Contact problems in elasticity", Soc. Ind. Appl. Math. (SIAM), Philadelphia, U. S. A., 1988.

[5] G. Lumer, "Semi-inner product spaces", Trans. Amer. Math. Soc. 100 (1961), 29-43.

[6] M. A. Noor, "General nonlinear variational inequalities", J. Math. Anal. Appl. 126 (1987), 78-84.

Mathematics Department, College of Science, King Saud University, P. O. Box 2455, Riyadh 11451, Saudi Arabia. 